\title{
Polymer Microring Coupled-Resonator Optical Waveguides
}

\author{
Joyce K. S. Poon, Student Member, IEEE, Lin Zhu, Guy A. DeRose, and Amnon Yariv, Life Fellow, IEEE
}

\begin{abstract}
We present measurements of the transmission and dispersion properties of coupled-resonator optical waveguides (CROWs) consisting of weakly coupled polymer microring resonators. The fabrication and the measurement methods of the CROWs are discussed as well. The experimental results agree well with the theoretical loss, waveguide dispersion, group delay, group velocity, and group-velocity dispersion (GVD). The intrinsic quality factors of the microrings were about $1.5 \times 10^{4}$ to $1.8 \times 10^{4}$, and group delays greater than $100 \mathrm{ps}$ were measured with a GVD between -70 and $100 \mathrm{ps} /(\mathrm{nm} \cdot$ resonator $)$. With clear and simple spectral responses and without a need for the tuning of the resonators, the polymer microring CROWs demonstrate the practicability of using a large number of microresonators to control the propagation of optical waves.
\end{abstract}

Index Terms-Microresonators, optical polymers, optical waveguides, periodic structures.

\section{INTRODUCTION}

$\mathbf{R}$ ESONATORS have the unique ability to store large amounts of energy built up from considerably weaker inputs. Because of their compact sizes, optical microresonators can trap this optical intensity in physically small volumes. Thus, we envision that chains of coupled microresonators may provide a new method for reducing the group velocity of optical pulses in a compact way on a chip. A coupledresonator optical waveguide (CROW) consists of a chain of coupled resonators in which light propagates by virtue of the coupling between adjacent resonators [1]-[3]. CROWs have the potential to significantly slow down the propagation and alter the dispersive properties of light, which may find applications such as optical delay lines, interferometers, optical buffers, and nonlinear optics [4]-[6].

For CROWs to be highly dispersive or to slow down light, a large number of weakly coupled identical resonators are required. However, the major challenge in realizing CROWs and using multiple resonators for dispersion engineering [7], [8] has been the fabrication of low-loss resonators with strict size tolerances. The problem is compounded when the resonators are weakly coupled because of the narrow linewidth of the coupled resonators. One solution is to tune the resonators individually, but controlling the tuning becomes more complicated

Manuscript received October 14, 2005; revised January 13, 2006. This work was supported by a scholarship from Natural Sciences and Engineering Research Council of Canada, Hughes Research Laboratories, The Defense Advanced Research Projects Agency, and the National Science Foundation.

The authors are with the Department of Electrical Engineering, California Institute of Technology (Caltech), Pasadena, CA 91125 USA (e-mail: poon@caltech.edu).

Digital Object Identifier 10.1109/JLT.2006.870971

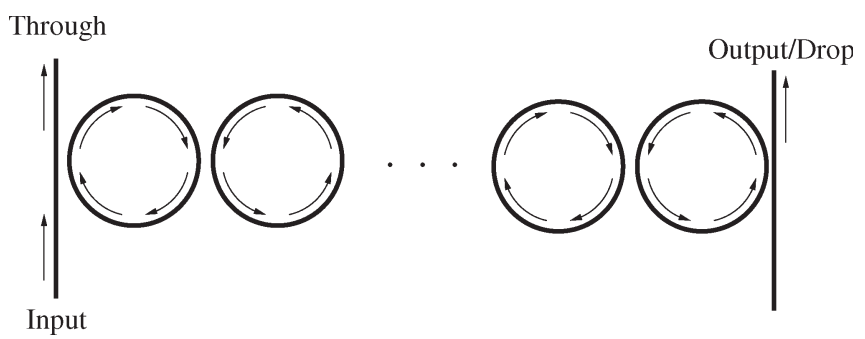

Fig. 1. Schematic of a finite microring CROW.

as the number of resonator increases. There have been several examples of high-order $(>10)$ coupled microresonators using microrings and photonic crystal defect cavities [6], [9]-[11]. However, the dispersion and delay were often not directly measured [6], [9], [10], and in the case of photonic crystal cavities, the resonators had low quality factors, and the transmission spectra may be quite complex [6], [11].

We have recently overcome some of the challenges in realizing CROWs and reported experimental measurements of the transmission and group delay in CROWs consisting of highorder weakly coupled polymer microring resonators [12]. In this paper, we describe in detail the fabrication and measurement of the CROWs as well as their transmission and dispersive properties. We shall address the issues of loss, ring resonator waveguide index and dispersion, group delay, and group velocity dispersion (GVD) in CROWs. Through comparisons between our experimental and theoretical results, we will verify a number of theoretically predicted properties of CROWs.

\section{THEORY}

We have previously analyzed ring resonator CROWs using transfer matrices [13] and derived a set of analytical expressions for the delay and loss of CROWs [14]. We shall briefly review these theoretical results, which will be useful in our comparisons with our experiments. Fig. 1 shows the schematic of a finite microring CROW with input and output waveguides. For phase-matched coupling, the sense of circulation of the wave alternates as the wave propagates along the chain of ring resonators.

As detailed in [13], the dispersion relation of a microring CROW is

$$
\sin (\beta \pi R)= \pm|\kappa| \cos (K \Lambda)
$$

where $\beta=n_{\text {eff }}(\omega) \omega / c$ is the propagation constant in the ring, $R$ is the radius, $\kappa=i|\kappa|$ is the dimensionless field coupling coefficient between two rings, $K$ is the Bloch wavevector, 
and $\Lambda$ is the periodicity of the structure. In the limit of weak coupling $|\kappa| \ll 1$, the dispersion relation reduces to

$$
\omega(K)=\Omega\left[1 \pm \frac{|\kappa|}{m \pi} \cos (K \Lambda)\right]
$$

where $\Omega$ is the resonance frequency of an uncoupled resonator in radians per second, and $m=\Omega n_{\mathrm{eff}}(\Omega) R / c$ is the azimuthal modal number.

Using (2), the group velocity $v_{\mathrm{g}}$ of a CROW, which is given by $1 / v_{\mathrm{g}}=\partial K / \partial \omega$, is

$$
\frac{1}{v_{\mathrm{g}}}=\frac{n_{\mathrm{eff}}}{\Lambda \sin (K \Lambda)}\left[ \pm \frac{R \pi}{c|\kappa|}-\frac{\cos (K \Lambda)}{n_{\mathrm{eff}}^{2}} \frac{\partial n_{\mathrm{eff}}}{\partial \omega}\right] .
$$

At the center of the CROW transmission band, where $\omega=\Omega$ and $K \Lambda=\pi / 2$, the magnitude of the group velocity is maximum and is equal to

$$
\left|v_{\mathrm{g}}(\Omega)\right|=\frac{c|\kappa| \Lambda}{\pi R n_{\mathrm{eff}}(\Omega)} .
$$

The time delay of a pulse propagating through the CROW $\tau$ is determined by the distance traversed in the CROW and the group velocity, such that

$$
\tau=\frac{N \Lambda}{v_{\mathrm{g}}}
$$

where $N$ is the number of resonators. At the center of the CROW band, the delay is equal to

$$
\tau_{\mathrm{d}}=\frac{N \pi R n_{\mathrm{eff}}(\Omega)}{c|\kappa|} .
$$

The loss of a CROW is given by the product of the time delay, the velocity of light in the resonators, and the loss per unit length in the resonators. At the center of the band, the loss $\alpha_{\Omega}$ is

$$
\alpha_{\Omega}=\frac{\alpha_{1} N \pi R}{|\kappa|}
$$

where $\alpha_{1}$ is the loss per length in the rings.

We shall define the slowing factor $S$ to be the ratio of the group velocity in a free space to the group velocity in the CROW, $S=c / v_{\mathrm{g}}$, such that at the band center

$$
S_{\Omega}=\frac{\pi n_{\mathrm{eff}}(\Omega)}{2|\kappa|} .
$$

Therefore, to obtain a large slowing factor, a weak interresonator coupling is necessary.

Using the conventional definition of the GVD, the GVD is given by the change of the delay time with respect to the wavelength [15]. Neglecting the dependence of $n_{\mathrm{eff}}$ on $\omega$, the GVD per resonator $D$ is

$$
D \equiv \frac{1}{N} \frac{\partial \tau}{\partial \lambda}=\frac{\Lambda^{3}(2 \pi c)^{2}}{v_{\mathrm{g}}^{3} \lambda^{2}}\left(\frac{1}{\lambda_{0}}-\frac{1}{\lambda}\right)
$$

1)

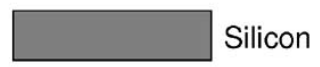

2)

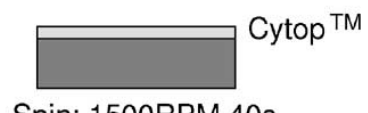

3)

Spin: 1500RPM 40s

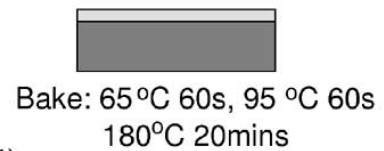

4) $180^{\circ} \mathrm{C} 20 \mathrm{mins}$

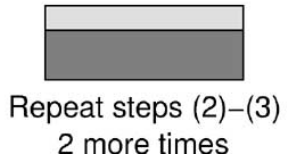

5)

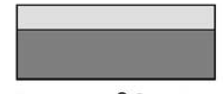

Bake: $180^{\circ} \mathrm{C} 3$ hours

6)

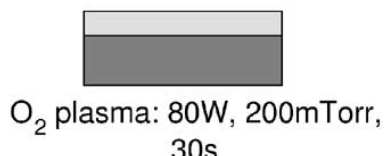

7) $30 \mathrm{~s}$

8)

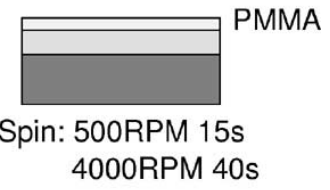

Bake: $180^{\circ} \mathrm{C} 20 \mathrm{mins}$
Fig. 2. Summary of the Cytop and PMMA preparation process for the electron-beam writing.

where $\lambda_{0}=2 \pi \Omega / c$ is the resonance wavelength. As evidenced by (9), the GVD is maximum at the band edges where $v_{\mathrm{g}} \rightarrow 0$ and minimum at the band center, where $\lambda=\lambda_{0}$. The GVD switches sign across the band center, such that for $v_{\mathrm{g}}>0$, it is negative for $\lambda<\lambda_{0}$ and positive for $\lambda>\lambda_{0}$.

From our spectral and delay measurements of CROWs, we shall verify (4)-(9) and determine the transmission and dispersive properties of the ring resonators.

\section{FABRICATION}

We fabricated CROWs with as many as 12 weakly coupled microring resonators in polymethyl methacrylate (PMMA) ( $n=1.49)$ by direct electron-beam writing. As the CROWs require numerous nearly identical resonators, PMMA is ideal for their fabrication since it is a high-resolution electron-beam resist. A low-index perfluoropolymer Cytop $(n=1.34$, Asahi Glass) was used as the lower cladding. The material system of PMMA and Cytop is used in commercial polymer optical fibers ${ }^{1}$ and has previously been used for simple waveguides [16]. The PMMA microrings did not have an upper cladding to keep the radius as small as possible.

Fig. 2 summarizes the Cytop and PMMA preparation process for the electron-beam writing. We began the fabrication process by depositing a $5.2 \mu \mathrm{m}$ thick layer of Cytop CTL-809M on a $250 \mu \mathrm{m}$ thick silicon substrate. To ensure flatness and uniformity over the wafer, the deposition of the Cytop was accomplished via a series of spinning and thermal curing steps. First, we spun the Cytop on the silicon at $1500 \mathrm{r} / \mathrm{min}$. Adhesion promoters were not necessary. Next, the Cytop was baked at $65{ }^{\circ} \mathrm{C}$ for $60 \mathrm{~s}, 95{ }^{\circ} \mathrm{C}$ for $60 \mathrm{~s}$, and $180{ }^{\circ} \mathrm{C}$ for $20 \mathrm{~min}$. The ramping of the bake temperature was critical in attaining flat

\footnotetext{
${ }^{1}$ E.g. Toray Raytela plastic optical fibers.
} 


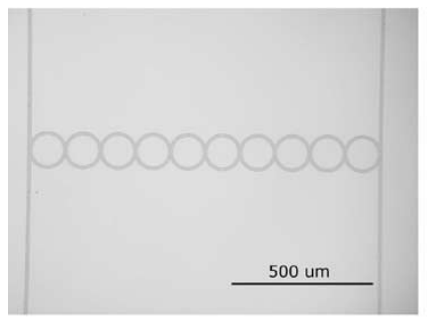

(a)

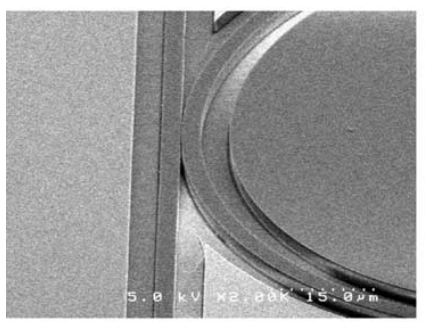

(c)

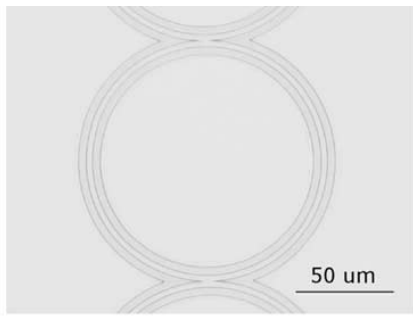

(b)

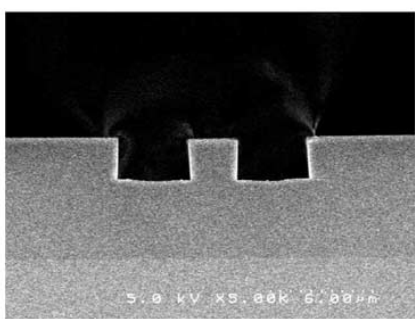

(d)
Fig. 3. Optical microscope $[(a),(b)]$ and scanning electron-microscope [(c), (d)] images of the fabricated devices in PMMA on Cytop on silicon. (a) Ten coupled microring resonators. The ring radius is $60 \mu \mathrm{m}$. (b) One of the microrings and the coupler regions. (c) The coupling region between the input/output waveguide and the microring. (d) A waveguide end facet produced by cleaving.

and uniform surfaces. The spinning and baking steps were then repeated two more times, with a final bake at $180^{\circ} \mathrm{C}$ for $3 \mathrm{~h}$.

After the chip cooled down, an oxygen plasma treatment of the Cytop was necessary for the adhesion of Cytop to PMMA. The plasma exposure was $30 \mathrm{~s}$ long at an RF power of $80 \mathrm{~W}$ and $\mathrm{O}_{2}$ pressure of $200 \mathrm{mtorr}$. After an optional $60 \mathrm{~s}$ exposure to hexamethyldisilazane (HMDS), a $2.6 \mu \mathrm{m}$ of PMMA 950K C10 (Microchem) was spun onto the chip at $500 \mathrm{r} / \mathrm{min}$ for $15 \mathrm{~s}$ and then $4000 \mathrm{r} / \mathrm{min}$ for $40 \mathrm{~s}$. A pre-exposure bake at $180{ }^{\circ} \mathrm{C}$ for 20 min ensured solvents were evaporated and improved the adhesion between the Cytop and PMMA.

We next patterned the microrings via direct electron-beam writing (Leica EBPG5000). Since PMMA is a positive resist, we defined the cladding regions with the electron-beam lithography. We used an acceleration voltage of $100 \mathrm{kV}$ and an electron-beam current of $3.5 \mathrm{nA}$ at a dosage varying from 785 to $815 \mu \mathrm{C} / \mathrm{cm}^{2}$. After the electron-beam exposure, we developed the sample in a 1:3 methyl isobutyl ketone (MIBK): isopropanol (IPA) solution. Finally, we separated the devices by cleaving.

Fig. 3 shows several optical and scanning electronmicroscope pictures of the fabricated devices. The electronbeam lithography produced waveguides with fairly smooth sidewalls. Fig. 3(d) shows the device end facet, which was defined by scribing and breaking. The slight waviness of the Cytop near the PMMA waveguide is an artifact of charging during the scanning electron-microscope imaging. The quality of the end facet indicates a good adhesion between the PMMA and Cytop and between the Cytop and silicon. It also shows that both PMMA and Cytop possess the mechanical properties suitable to cleaving. The waveguides had a width of $2.9 \mu \mathrm{m}$ and a height of $2.6 \mu \mathrm{m}$. The cladding regions were $4 \mu \mathrm{m}$ wide. The radius of the rings was $60 \mu \mathrm{m}$ such that the bend loss, as calculated using a radial beam propagation method, would be $<1 \mathrm{~dB} / \mathrm{cm}$. There was no coupling gap between the resonators

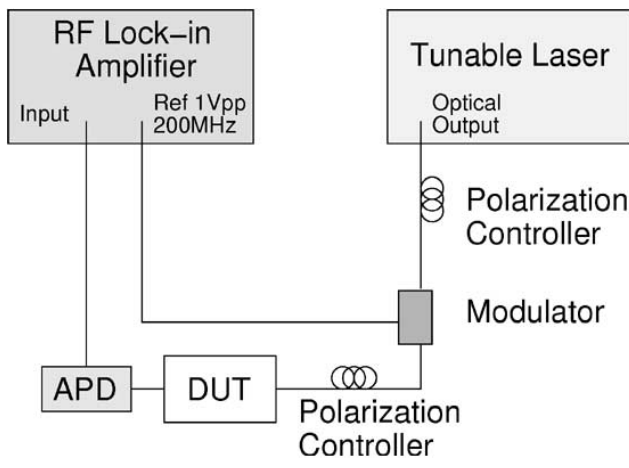

Fig. 4. Schematic of the group delay measurement setup. The RF lockin amplifier generates a $1-\mathrm{V}$ peak-to-peak voltage at $200 \mathrm{MHz}$ to drive the modulator. DUT is the device under test and APD is the avalanche photodiode.

and between the waveguide and first/last resonator. However, due to the radius of curvature of the rings as well as the waveguide design and index contrast, even without a coupling gap, weak coupling between the resonators was achieved.

\section{Transmission and Group Delay Measurements}

We measured both the transmission spectra and group delays of the fabricated microring CROWs. The spectral measurements were straightforward wherein we detected the transmitted output power as a function of the wavelength scanned by a tunable laser. The group delay measurement was performed using an RF phase-shift technique [17], [18].

Fig. 4 is a schematic of the setup of the group delay measurement. An RF lock-in amplifier (SR844) generated the drive voltage to a modulator and detected the phase shift between the drive and measured signals. Light from the tunable laser source was coupled into the device under test (DUT) via a single-mode fiber. The transmitted light was collected with a multimode fiber coupled to a high-speed $(2.5 \mathrm{GHz})$ InGaAs avalanche photodiode (APD). To determine the absolute time delay through the CROW, we measured the reference phase shift due to the propagation through the input and output waveguides only and calibrated for any intrinsic intensity dependent system response. Thus, the measured group delay through the CROW is given by

$$
\tau_{\mathrm{m}}=\frac{\theta_{\mathrm{m}}-\theta_{\mathrm{ref}}}{360^{\circ}} \frac{1}{f_{\mathrm{mod}}}
$$

where $\theta_{\mathrm{m}}$ is the measured phase-shift angle in degrees, $\theta_{\text {ref }}$ is the reference angle in degrees, and $f_{\text {mod }}$ is the modulation frequency in Hertz. By changing the wavelength of the tunable laser source, we measured the group delay as a function of the optical frequency.

The accuracy of the group delay depends on the accuracy of the measured phase shift. For a fixed error in the measured angle, the error in the group delay is smaller for a higher modulation frequency by (10). However, a high modulation frequency may cause significant distortions in the delay and amplitude measurements with the lock-in amplifier, because the two sidebands generated about the optical carrier may 


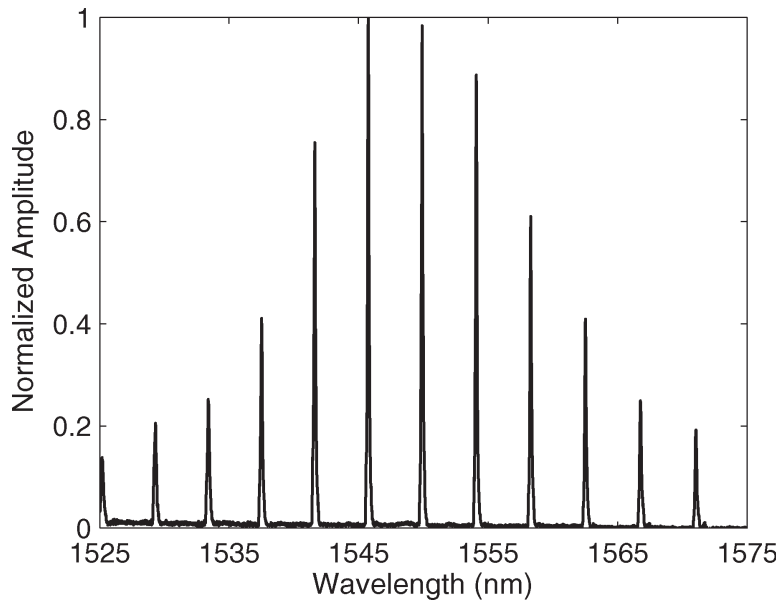

Fig. 5. Drop port transmission spectrum of TE polarized light through a CROW of 10 coupled microring resonators.

experience vastly different transmission characteristics in a narrowband device. This distortion is less pronounced if $f_{\mathrm{mod}}$ is kept significantly smaller than the bandwidth of the device [17]. The measured angle can also have an ambiguity equal to multiples of $360^{\circ}$, equivalent to a delay of $1 / f_{\bmod }$. Hence, a higher modulation frequency would more easily lead to uncertainty in the group delay due to possible $360^{\circ}$ phase shifts.

For our experiments, we used a modulation frequency of $200 \mathrm{MHz}$, which was about 100 times narrower than the fullwidth at half-maximum (FWHM) linewidth of the CROWs. A maximum delay of $5 \mathrm{~ns}$ can be measured at this modulation frequency without encountering the $360^{\circ}$ ambiguity. However, the phase error in our measurement was about $\pm 0.5^{\circ}$, equivalent to $\mathrm{a} \pm 7 \mathrm{ps}$ uncertainty in the time delay. The tradeoff between the modulation frequency and the accuracy of the group delay is intrinsic to this measurement technique.

\section{Magnitude Response}

\section{A. Transmission Spectra}

Fig. 5 shows the transmission spectrum at the drop port of a 10 microring long CROW for TE polarized light. There are no spurious peaks in the spectrum, indicating that the resonators were nearly identical. However, slight variations in the resonators and polarization mixing may have caused the broad envelope in the spectrum.

We compared our measured results with the theoretical results computed from the transfer matrices [13]. For the theoretical calculations, we assumed the resonators to be identical and neglected the dependence of $n_{\text {eff }}$ on the wavelength. The lineshapes of both the drop and through ports are sensitive to the propagation loss in the rings, but while the drop port is sensitive to the interresonator coupling, the through port is more sensitive to the coupling between the input/output waveguides and the rings. Therefore, by fitting the drop and through spectra as well as the group delay, we could estimate the complete set of parameters that describe an ideal CROW composed of identical resonators: the propagation loss in the resonators, the interresonator coupling coefficient, and the waveguideresonator coupling coefficient.

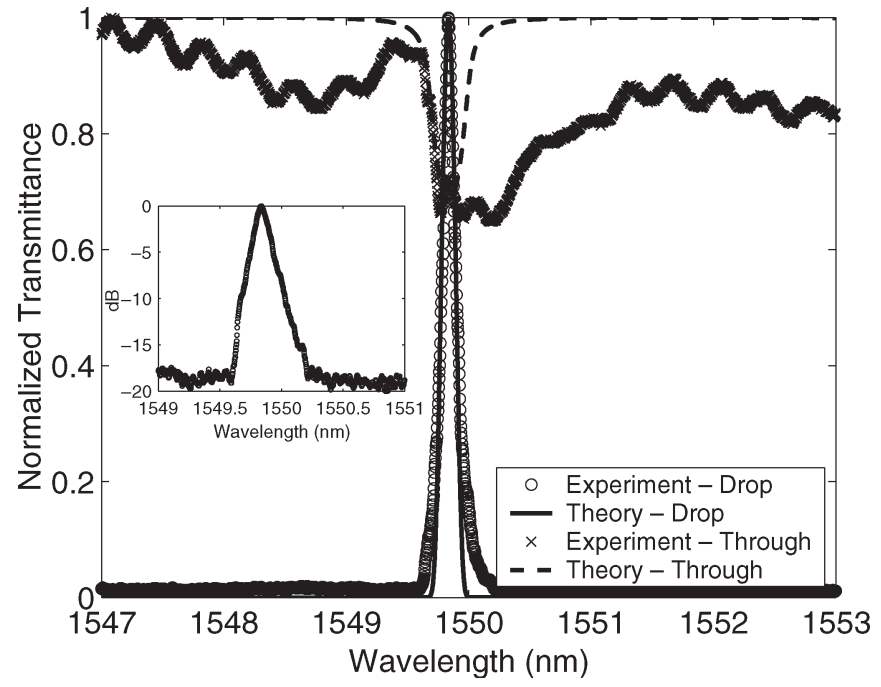

Fig. 6. Experimental and theoretical spectra at the drop and through ports for the transmission peak near $\lambda=1550 \mathrm{~nm}$ in Fig. 5. The fit parameters are $|\kappa|=0.12,|\kappa|_{\text {wg }}=0.15$, and $\alpha_{1}=17 \mathrm{~dB} / \mathrm{cm}$. Inset: The measured drop port spectrum in decibel scale.

Fig. 6 shows the experimentally measured spectra at the drop and through ports at the resonance near $1550 \mathrm{~nm}$ in Fig. 5 . The inset shows the drop port spectrum in decibel scale. The measured extinction ratio of circa $-20 \mathrm{~dB}$ was limited by the noise floor of our detector. For the fit, the interresonator field coupling coefficient is $|\kappa|=0.12$, the waveguideresonator field coupling coefficient is $|\kappa|_{\mathrm{wg}}=0.15$, and the propagation loss is $17 \mathrm{~dB} / \mathrm{cm}$. The through port spectrum shows the Fabry-Pérot resonances defined by the device end facets. The multiple notches in the spectrum indicate there were indeed variations in the resonators, which were not as apparent in the lineshape of the drop port. However, these variations were small enough such that we were able to obtain simple, clear spectral responses, as in Fig. 5. The ring resonators were under coupled to the input waveguide so that the extinction of the notch in Fig. 6 is only about $-1.5 \mathrm{~dB}$.

\section{B. Losses}

In general, the CROWs we fabricated had interresonator coupling coefficients $|\kappa|$ of about 0.1 to 0.15 . The propagation losses of the ring resonators were about 15 to $18 \mathrm{~dB} / \mathrm{cm}$, resulting in intrinsic quality factors of $1.5 \times 10^{4}$ to $1.8 \times 10^{4}$. Most of the propagation loss was likely due to sidewall scattering since the index contrast between the core and the air cladding was quite large. The material losses of PMMA are about 1.5 to $2 \mathrm{~dB} / \mathrm{cm}$ [19], [20], and the theoretical bend loss was less than $1 \mathrm{~dB} / \mathrm{cm}$. Due to the losses in the CROWs, ripples in the passband were not observed. Passband ripples can introduce distortions to and limit the bandwidth of propagating optical pulses [4], [17]. The ripples may be reduced by choosing a suitable waveguide-resonator coupling coefficient [14], or the passband can be optimally flattened through the apodization of the interresonator coupling coefficients [4], [17], [21].

The fiber-to-fiber insertion loss at the through port was about -15 to $-20 \mathrm{~dB}$ OFF-resonance, and for ON-resonance, the loss was about -16 to $-21 \mathrm{~dB}$. The fiber-to-fiber insertion loss 


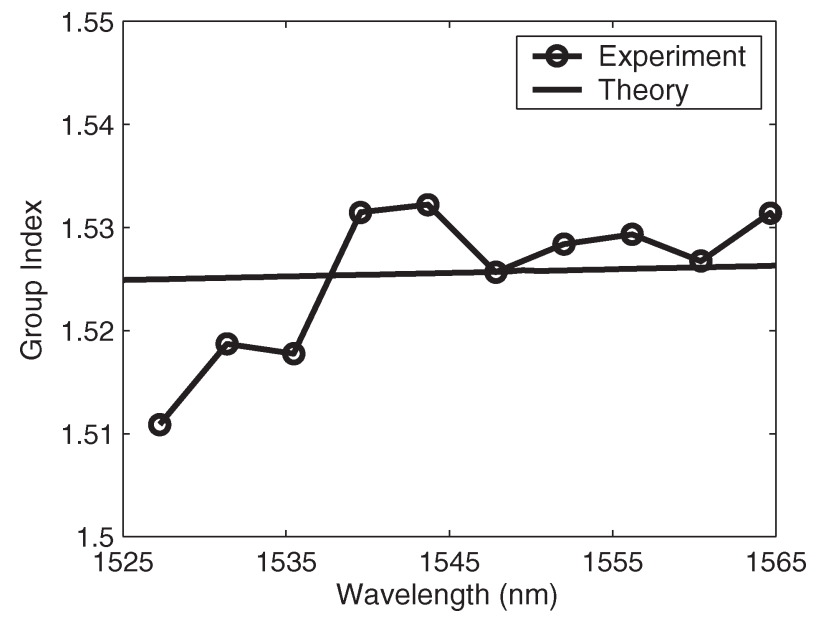

Fig. 7. Experimentally measured and theoretically calculated group index for the PMMA on Cytop ring resonators. The experimental values are extrapolated from the spectrum in Fig. 5.

at the drop port depends on the number of resonators in the CROW and varied from about $-35 \mathrm{~dB}$ for four resonators to $-45 \mathrm{~dB}$ for 12 resonators. The ratio of the drop power to the difference between the ON and OFF resonance through power gives equivalent losses of 2.4 to $3.5 \mathrm{~dB}$ per resonator, which is in excellent agreement with the loss of 2.3 to $3 \mathrm{~dB}$ per resonator calculated with (7).

The measured spectrum and loss per resonator suggest that while slight variations in the resonators existed, the microrings comprising the CROWs were nearly identical. Our results show that the maximum number of microrings that can be coupled together is not limited by the fabrication accuracy but rather by the resonator losses.

\section{DISPERSIVE PROPERTIES}

To comprehensively characterize the CROWs, we obtained the dispersive properties of the ring resonators and the CROW as well. In this section, we shall extrapolate the group and effective indices of resonator waveguides, the group delay and slowing factors in the CROWs, and the CROW GVD from our spectral and delay measurements.

\section{A. Group Index}

The group index of the resonator waveguides $n_{\mathrm{g}}$ is related to the free spectral range of the resonator

$$
\Delta f_{\mathrm{FSR}}=\frac{c}{2 \pi n_{\mathrm{g}} R}
$$

where $\Delta f_{\mathrm{FSR}}$ is the free spectral range in frequency, and the group index is defined as

$$
n_{\mathrm{g}}\left(\lambda_{1}\right)=n_{\mathrm{eff}}\left(\lambda_{1}\right)-\lambda_{1} \frac{\partial n_{\mathrm{eff}}}{\partial \lambda}{ }_{\mid \lambda_{1}} .
$$

Therefore, from the transmission spectrum, we may obtain the group index as a function of the wavelength. Fig. 7 shows the group index extrapolated from the transmission spectrum in Fig. 5. We have also plotted the theoretical group index of
TABLE I

COUPLING COEFFICIENTS, GROUP DELAYS, AND SLOWING FACTORS FOR CROWS OF VARIOUS LENGTHS

\begin{tabular}{cccc}
\hline $\begin{array}{c}\text { Number of } \\
\text { Microrings }(\mathbf{N})\end{array}$ & $\begin{array}{c}\text { Coupling } \\
|\kappa|\end{array}$ & $\begin{array}{c}\text { Group Delay } \\
\tau_{\mathbf{d}}(\mathrm{ps})\end{array}$ & $\begin{array}{c}\text { Slowing Factor } \\
\left(\mathbf{S}_{\boldsymbol{\Omega}}\right)\end{array}$ \\
\hline 4 & 0.15 & 25.9 & 16.2 \\
6 & 0.25 & 23 & 9.6 \\
8 & 0.15 & 49 & 15.2 \\
10 & 0.12 & 80 & 19.9 \\
12 & 0.10 & 110 & 22.9 \\
\hline
\end{tabular}

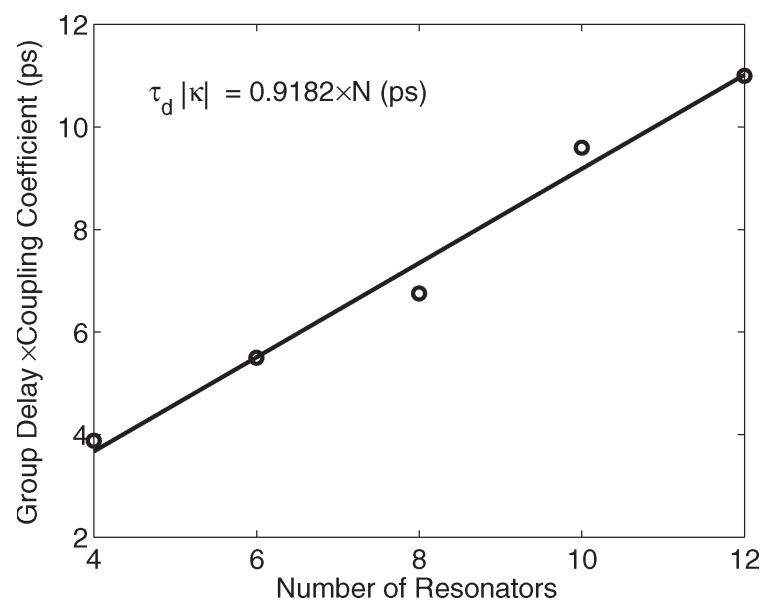

Fig. 8. Product of the time delay at band center and the interresonator coupling $\tau_{\mathrm{d}}|\kappa|$ is plotted against the number of resonators using the results summarized in Table I. The slope of the graph gives $\pi R n_{\mathrm{eff}} / c$ according to (6).

the ring resonators calculated using a mode-solver. There is generally good agreement between the theoretical and experimental values. The calculated group index is approximately 1.525 and the measured group index ranges from 1.51 to 1.53 . The variation in the measured group index may be due to slight inaccuracies in the wavelength and material dispersion, which was not accounted for in the mode-solver calculations.

\section{B. Effective Index and Group Delay}

The effective index $n_{\text {eff }}$ from the mode-solver calculations is approximately 1.42 in this wavelength range. We may also obtain the effective index by using (6), since the group velocity at the center of the CROW band depends on the effective index and not the group index. Table I lists the interresonator coupling coefficients, measured group delays, and slowing factors for TE polarized light in CROWs of various lengths that were fabricated. By plotting $\tau_{\mathrm{d}}|\kappa|$ versus $N$, according to (6), the slope is proportional to the effective index averaged over the wavelength range considered. Thus, we can compare the experimental effective index with the theoretical value and verify (6).

Fig. 8 shows the $\tau_{\mathrm{d}}|\kappa|$ as a function of $N$. The data fits very well with a linear function, with a slope of $0.9182 \mathrm{ps}$, translating to an effective index of 1.46. The value agrees with the calculated value of 1.42 within the experimental error, indicating that (6) accurately expresses the time delay at the band center of a CROW.

As listed in Table I, the resonators in the CROWs were generally weakly coupled, with an interresonator intensity coupling of about $1 \%$ to $2 \%$. The weak interresonator coupling led to 


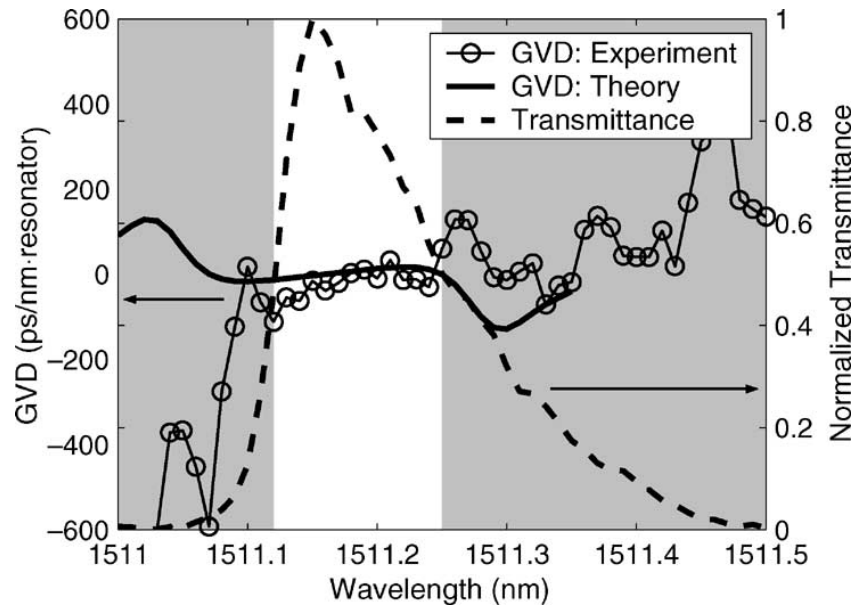

Fig. 9. GVD of TE polarized light in a 12-microring-long CROW with delay properties listed in Table I. The transmittance of the resonance is plotted for reference. The unshaded region is within the FWHM of the transmission peak.

slowing factors at the maximum of the transmission peaks of about 15 to 25 . The FWHM of the transmission peaks was approximately 15 to $20 \mathrm{GHz}$. The coupling coefficient was highly sensitive to the electron-beam writing conditions and the PMMA/Cytop layers such that the coupling coefficient was not replicated exactly from device to device. Nonetheless, as evidenced by Fig. 8, the group delays of the devices were consistent with each other.

\section{GVD}

Finally, we can determine the GVD of the CROW from the group delay measurements. Theoretically, the GVD is given by (9). The GVD switches sign across the resonance frequency $\Omega$ and is highest at the band edges where the group velocity is small. We extracted the GVD by taking the derivative of the measured group delay with respect to the wavelength.

Fig. 9 shows the per resonator GVD for the TE polarization of a CROW consisting of 12 microrings, for which the delay properties are listed in Table I. The normalized transmittance is also shown for reference. The unshaded region is within the FWHM of the transmission peak. The curvature of the theoretically calculated GVD changes at the band edges due to the losses in the resonators [22]. The measured GVD follows the general trend described by (9). In Fig. 9, the GVD changes from negative to positive across the resonance peak. The high GVD at the edges of the peak may not be physical, since the transmission amplitude was low at these wavelengths. The change in the GVD curvature at the band edges in the calculated result could not be measured, most likely because of the lowtransmission amplitude.

Unsurprisingly, the GVD of the CROW can be very high. The measured GVD varied from -100 to $70 \mathrm{ps} /(\mathrm{nm} \cdot$ resonator $)$ across the FWHM of the peak, with zero GVD at $1511.18 \mathrm{~nm}$, near the resonance peak at $1511.15 \mathrm{~nm}$. The measured GVD is significantly higher than the theoretically calculated GVD, which ranges from -17 to $17 \mathrm{ps} /(\mathrm{nm} \cdot$ resonator $)$ across the FWHM of the transmission peak. The dispersion of the res- onator waveguide alone does not account for the difference. The per resonator GVD due to waveguide dispersion is

$$
D_{\mathrm{wg}}=\frac{\pi R}{c|\kappa|} \frac{\partial n_{\mathrm{g}}}{\partial \lambda}
$$

which is approximately $2.2 \times 10^{-4} \mathrm{ps} /(\mathrm{nm} \cdot$ resonator $)$. The discrepancy may be a result of the deviation from the ideal scenario of identical resonators. The asymmetry of the transmission peak suggests the resonators were not perfectly identical and perhaps the polarization was not purely TE. Since the GVD, given by (9), scales as $1 / v_{\mathrm{g}}^{3}$, any slight deviation of the group velocity will result in a large change in the dispersion.

Compared to other engineered waveguide structures reported to date, such as photonic crystal waveguides and fibers, the CROWs we have demonstrated possess a significantly higher GVD, even though the refractive indices of the polymer materials are relatively low. The measured GVD values of about $\pm 100 \mathrm{ps} /(\mathrm{nm} \cdot$ resonator $)$ are equivalent to $\pm 8.3 \times$ $10^{8} \mathrm{ps} /(\mathrm{nm} \cdot \mathrm{km})$, and the calculated GVD of $\pm 17 \mathrm{ps} /(\mathrm{nm}$. resonator) is equivalent to $\pm 1.4 \times 10^{8} \mathrm{ps} /(\mathrm{nm} \cdot \mathrm{km})$. The CROWs we have presented are about $10^{7}$ times more dispersive than conventional optical fibers, $10^{6}$ times more dispersive than highly dispersive photonic crystal fibers [23], and approximately 100 to 1000 times more dispersive than photonic crystal waveguides reported to date [24], [25]. Compared to previously reported GVD values of photonic crystal CROWs [26], the GVD of our microring CROWs is about an order of magnitude greater. With such large values of both normal and anomalous dispersion, CROWs may find applications in dispersion management and nonlinear optics [15], [27]-[31].

\section{CONCLUSION}

We have fabricated microring CROWs in polymer materials and have measured their spectral and dispersive properties. Direct electron-beam writing of the PMMA on the Cytop produces nearly identical microrings such that no external tuning of the ring resonators is necessary, greatly simplifying the fabrication and characterization process. The simple and clear transmission spectra of the microring CROWs are in sharp contrast to other resonators such as disks, spheres, and photonic crystal defects. The maximum number of coupled rings and the maximum achievable delay are limited by the loss in the resonators and not by any fabrication inaccuracies. Group delays greater than $100 \mathrm{ps}$ were measured in the CROWs, with slowing factors of circa 15 to 25 . The GVD of the CROWs can be very high [about $\pm 100 \mathrm{ps} /(\mathrm{nm} \cdot$ resonator)], with most of the dispersion arising from the CROW device structure rather than from the material or waveguide dispersion. Our demonstration illustrates the feasibility of using a large number of microresonators to engineer the transmission and dispersion of optical waves.

\section{ACKNOWLEDGMENT}

The authors would like to thank Prof. A. Scherer for the use of his fabrication facilities. J. Poon thanks Dr. Y. Huang, Dr. R. K. Lee, J. Choi, and Dr. W. M. J. Green for helpful discussions. 


\section{REFERENCES}

[1] A. Yariv, Y. Xu, R. K. Lee, and A. Scherer, "Coupled-resonator optical waveguide: A proposal and analysis," Opt. Lett., vol. 24, no. 11, pp. 711-713, Jun. 1999.

[2] N. Stefanou and A. Modinos, "Impurity bands in photonic insulators," Phys. Rev. B, Condens. Matter, vol. 57, no. 19, pp. 12127-12133, May 1998.

[3] A. Melloni and F. Morichetti, "Linear and nonlinear pulse propagation in coupled resonator slow-wave optical structures," Opt. Quantum Electron., vol. 35, no. 4/5, pp. 365-379, Mar. 2003.

[4] G. Lenz, B. J. Eggleton, C. K. Madsen, and R. E. Slusher, "Optical delay lines based on optical filters," IEEE J. Quantum Electron., vol. 37, no. 4, pp. 525-532, Apr. 2001.

[5] S. Lan, S. Nishikawa, H. Ishikawa, and O. Wada, "Engineering photonic crystal impurity bands for waveguides, all-optical switches and optical delay lines," IEICE Trans. Electron., vol. E85C, no. 1, pp. 181-189, 2002.

[6] H. Altug and J. Vuckovic, "Experimental demonstration of the slow group velocity of light in two-dimensional coupled photonic crystal microcavity arrays," Appl. Phys. Lett., vol. 86, no. 11, p. 111102 , Mar. 2005.

[7] J. E. Heebner and R. W. Boyd, "Slow' and 'fast' light in resonatorcoupled waveguides," J. Mod. Opt., vol. 49, no. 14/15, pp. 2629-2636, 2002.

[8] L. Maleki, A. B. Matsko, A. A. Savchenkov, and V. S. Ilchenko, "Tunable delay line with interacting whispering-gallery-mode resonators," Opt. Lett., vol. 29, no. 6, pp. 626-628, Mar. 2004

[9] B. E. Little, S. T. Chu, P. P. Absil, J. V. Hryniewicz, F. G. Johnson, F. Seiferth, D. Gill, V. Van, O. King, and M. Trakalo, "Very highorder microring resonator filters for WDM applications," IEEE Photon. Technol. Lett., vol. 16, no. 10, pp. 2263-2265, Oct. 2004.

[10] T. D. Happ, M. Kamp, A. Forchel, J. L. Gentner, and L. Goldstein, "Two-dimensional photonic crystal coupled-defect laser diode," Appl. Phys. Lett., vol. 82, no. 1, pp. 4-6, Jan. 2003.

[11] S. Nishikawa, S. Lan, N. Ikeda, Y. Sugimoto, H. Ishikawa, and K. Asakawa, "Optical characterization of photonic crystal delay lines based on one-dimensional coupled defects," Opt. Lett., vol. 27, no. 23, pp. 2079-2081, Dec. 2002.

[12] J. K. S. Poon, L. Zhu, G. A. DeRose, and A. Yariv, "Transmission and group delay in microring coupled-resonator optical waveguides," Opt. Lett., vol. 31, no. 4, pp. 456-458, 2006.

[13] J. K. S. Poon, J. Scheuer, S. Mookherjea, G. T. Paloczi, Y. Huang, and A. Yariv, "Matrix analysis of microring coupled-resonator optical waveguides," Opt. Express, vol. 12, no. 1, pp. 90-103, Jan. 2004.

[14] J. K. S. Poon, J. Scheuer, Y. Xu, and A. Yariv, "Designing coupledresonator optical waveguide delay lines," J. Opt. Soc. Amer. B, Opt. Phys., vol. 21, no. 9, pp. 1665-1673, Sep. 2004.

[15] S. Mookherjea, "Dispersion characteristics of coupled-resonator optical waveguides," Opt. Lett., vol. 30, no. 18, pp. 2406-2408, Sep. 2005.

[16] Y.-G. Zhao, W.-K. Lu, Y. Ma, S.-S. Kim, and S. T. Ho, "Polymer waveguides useful over a very wide wavelength range from the ultraviolet to infrared," Appl. Phys. Lett., vol. 77, no. 19, pp. 2961-2963, Nov. 2000 .

[17] C. K. Madsen and J. H. Zhao, Optical Filter Design and Analysis: A Signal Processing Approach. New York: Wiley, 1999.

[18] A. Stapleton, S. Farrell, H. Akhavan, R. Shafiiha, Z. Peng, S.-J. Choi, J. O'Brien, and P. D. Dapkus, "Optical phase characterization of active semiconductor microdisk resonators in transmission," Appl. Phys. Lett., vol. 88, no. 3, p. 31106, Jan. 2006.

[19] Y. Q. Shi, C. Zhang, H. Zhang, J. H. Bechtel, L. R. Dalton, B. H. Robinson, and W. H. Steier, "Low (sub-1-volt) halfwave voltage polymeric electro-optic modulators achieved by controlling chromophore shape," Science, vol. 288, no. 5463, pp. 119-122, Apr. 2000.

[20] S. L. Logunov, "Cavity ringdown detection of losses in thin films in the telecommunication wavelength window," Appl. Opt., vol. 40, no. 9, pp. 1570-1573, Mar. 2001.

[21] B. E. Little, S. T. Chu, H. A. Haus, J. Foresi, and J.-P. Laine, "Microring resonator channel dropping filter," J. Lightw. Technol., vol. 15, no. 6, pp. 998-1005, Jun. 1997.

[22] H. Kogelnik and C. V. Shank, "Coupled-wave theory of distributed feedback lasers,” J. Appl. Phys., vol. 43, no. 5, pp. 2327-2335, May 1972.

[23] J. C. Knight, J. Arriaga, T. A. Birks, A. Ortigosa-Blanch, W. J. Wadsworth, and P. S. Russell, "Anomalous dispersion in photonic crystal fiber," IEEE Photon. Technol. Lett., vol. 12, no. 7, pp. 807-809, Jul. 2000.

[24] M. Notomi, K. Yamada, A. Shinya, J. Takahashi, C. Takahashi, and I. Yokohama, "Extremely large group-velocity dispersion of line-defect waveguides in photonic crystal slabs," Phys. Rev. Lett., vol. 87, no. 25, p. 253902 , Dec. 2001
[25] T. Asano, K. Kiyota, D. Kumamoto, B.-S. Song, and S. Noda, "Timedomain measurement of picosecond light-pulse propagation in a twodimensional photonic crystal-slab waveguide," Appl. Phys. Lett., vol. 84, no. 23, pp. 4690-4692, Jun. 2004.

[26] T. J. Karle, Y. J. Chai, C. N. Morgan, I. H. White, and T. F. Krauss, "Observation of pulse compression in photonic crystal coupled cavity waveguides," J. Lightw. Technol., vol. 22, no. 2, pp. 514-519, Feb. 2004.

[27] W. J. Kim, W. Kuang, and J. D. O'Brien, "Dispersion characteristics of photonic crystal coupled resonator optical waveguides," Opt. Express, vol. 11, no. 25, pp. 3431-3437, Dec. 2003.

[28] J. B. Khurgin, "Expanding the bandwidth of slow-light photonic devices based on coupled resonators," Opt. Lett., vol. 30, no. 5, pp. 513-515, Mar. 2005

[29] J. K. Ranka, R. S. Windeler, and A. J. Stentz, "Visible continuum generation in air-silica microstructure optical fibers with anomalous dispersion at 800 nm," Opt. Lett., vol. 25, no. 1, pp. 25-27, Jan. 2000.

[30] S. Mookherjea and A. Yariv, "Kerr-stabilized super-resonant modes in coupled-resonator optical waveguides," Phys. Rev. E, vol. 66, no. 4 p. 46610 , Oct. 2002.

[31] D. N. Christodoulides and N. K. Efremidis, "Discrete temporal solitons along a chain of nonlinear coupled microcavities embedded in photonic crystals," Opt. Lett., vol. 27, no. 8, pp. 568-570, Apr. 2002.

Joyce K. S. Poon (S'02) received the B.A.Sc. degree in engineering science (physics option) from the University of Toronto, Toronto, ON, Canada, and the M.S. degree in electrical engineering from the California Institute of Technology (Caltech), Pasadena, in 2002 and 2003, respectively. She is working toward the $\mathrm{Ph} . \mathrm{D}$. degree in electrical engineering at Caltech.

Her research interests include periodic photonic structures, optical resonators, and guided-wave optics.

Lin Zhu received the B.Eng. and M.S. degrees in electrical engineering from Tsinghua University, Beijing, China, in 2000 and 2002, respectively. He also received the M.S. degree in electrical engineering from California Institute of Technology (Caltech), Pasadena, in 2004. He is working toward the Ph.D. degree in electrical engineering at Caltech.

His research interests include semiconductor lasers, periodic photonic structures, and optical resonators.

Guy A. DeRose received the B.S. degree from Indiana University, Indiana, PA, and the M.S. and Ph.D. degrees, where he studied thin-film mechanical properties via X-ray absorption spectroscopy, from Case Western Reserve University, Cleveland, OH, in 1987, 1989, and 1992, respectively, all in physics.

$\mathrm{He}$ has been the Laboratory Coordinator of the nanofabrication group at California Institute of Technology (Caltech), Pasadena, since 2000, where he studies integration of optics with microfluidics and physical properties and fabrication of nanometer-scale wires. He is also Co-Director of Caltech's LargeScale Integration of Nanostructures Laboratory, in which he is performing research on electron beam lithography.

Amnon Yariv (S'56-M'59-F'70-LF'95) received the B.S., M.S., and Ph.D. degrees in electrical engineering from the University of California, Berkeley, in 1954, 1956, and 1958, respectively.

In 1959, he joined Bell Telephone Laboratories, Murray Hill, NJ. In 1964, he joined the California Institute of Technology (Caltech), Pasadena, as an Associate Professor of electrical engineering, becoming a Professor in 1966. In 1980, he became the Thomas G. Myers Professor of electrical engineering and applied physics. In 1996, he became the Martin and Eileen Summerfield Professor of applied physics and Professor of electrical engineering. On the technical and scientific sides, he took part (with various co-workers) in the discovery of a number of early solid-state laser systems, in the original formulation of the theory of nonlinear quantum optics; in proposing and explaining mode-locked ultrashort-pulse lasers, GaAs optoelectronics; in proposing and demonstrating semiconductor-based integrated optics technology; in pioneering the field of phase conjugate optics; and in proposing and demonstrating the semiconductor distributed feedback laser. He has published widely in the laser and optics fields and has written a number of basic texts in quantum electronics, optics, and quantum mechanics.

Dr. Yariv is a member of the American Academy of Arts and Sciences, the National Academy of Engineering, and the National Academy of Sciences. 